ISSN 0001-6002/2003/45/3/107-112

Acta Médica Costarricense,(@2003

Colegio de Médicos y Cirujanos

\title{
Leptina, lípidos y sobrepeso en escolares de sexto grado de un área urbano-marginal
}

\author{
Laclé- Adriana ', Takayuki- Tamada ${ }^{1}$, Serrú- Lisseth ${ }^{2}$, Salas- Pilar ${ }^{~}$, Martínez- Virya ${ }^{3}$, Salas- Edwin ${ }^{3}$.
}

\begin{abstract}
Introducción: La hormona llamada Leptina ha sido asociada con varios factores del Síndrome metabólico, incluyendo hipertensión arterial, obesidad, resistencia insulínica y lipoproteína de alta densidad no solo en adultos, también en adolescentes. También en el modelo animal se ha relacionado con la diabetes mellitus. En Costa Rica no se conocen sus niveles plasmáticos, ni su relación con la obesidad y lípidos en adolescentes.
\end{abstract}

Objetivo: Investigar los niveles de leptinemia en escolares de sexto grado de un área urbano-marginal, y su asociación con lípidos y sobrepeso.

Material y métodos: Estudio transversal realizado año 2001, con muestra aleatoria de escolares de sexto grado de 12 escuelas del Área de Desamparados $3(\mathrm{n}=220)$. Previo consentimiento escrito de padres y escolares se midió peso y talla; se obtuvo suero en ayunas de 12 horas para cuantificar leptina (IRMA), colesterol total,HDLcolesterol y triglicéridos; el LDL-col se calculó.

Resultados: Los niveles de leptinemia mostraron una distribución no normal, por lo cual su análisis se realizó con métodos no paramétricos. Su rango varió desde una mínima de $1.0 \mathrm{ng} / \mathrm{ml}$, a un valor máximo de $42.8 \mathrm{ng} / \mathrm{ml}$ en el sexo masculino, y de $3.9 \mathrm{ng} / \mathrm{ml}$ a $65.2 \mathrm{ng} / \mathrm{ml}$ en el sexo femenino. La comparación de cuartiles según sexo fue altamente significativa ( $\mathrm{p}<0.0003$ ) siendo mayor en las mujeres. La concentración de leptina en plasma presentó una correlación positiva aunque baja con el Índice de Masa Corporal (IMC) ( $\mathrm{rs}=0.43$, p <5.7 E-11) y los triglicéridos $(\mathrm{rs}=0.31, \mathrm{p}<4.3 \mathrm{E}-06)$. El HDL-colesterol presentó también una baja correlación pero negativamente ( $r s-0.20, p<0.002)$. No se encontró correlación con colesterol total, ni LDL-colesterol. Al desglosar esto por sexo, esta correlación positiva con el IMC fue dada sobretodo por el sexo femenino (rs 0.59, p < 1.05E-12) y ésta fue moderada. . El comportamiento de los triglicéridos fue similar en ambos sexos, no así en el HDL, en donde la correlación negativa solo se presentó en las mujeres (rs - $0.40, \mathrm{p}<5.1 \mathrm{E}-06$ ), pero también fue baja. Al comparar los cuartiles de los valores de leptina contra el estado nutricional de los niños, se encontró diferencias significativas para ambos sexos entre las categorías de normal y sobrepeso $(\mathrm{p}<0.01)$ delgado y sobrepeso $(<0.05)$, no así entre delgado y normal.

Conclusión: Los valores de leptinemia en escolares fueron mayores en el sexo femenino. La correlación de esta variable aunque débil, fue positiva con IMC y negativa con HDL sobretodo en mujeres. Se encontraron diferencias significativas entre los niños delgado/normales y los que tenían sobrepeso. Los hallazgos de este estudio son coincidentes con los descritos en la literatura internacional.

Descriptores: Leptina, lípidos, sobrepeso, adolescentes

Recibido: 28 de febrero, 2003

Aceptado: 22 de julio, 2003

Abreviaturas: DM, Diabetes mellitus; IMC, índice de masa corporal.

1. Instituto de Investigaciones en Salud, Universidad de Costa Rica (INISA -UCR)

2. Nutrición, Área de Salud Desamparados 3 C.C.S.S.

3. Laboratorio Clínico, Clínica Dr. Marcial Fallas, Desamparados, C.C.S.S.

Correspondencia: Adriana Laclé Murray, Apdo. 18-2400 Desamparados, Correo electrónico:alacle@ cariari.ucr.ac.cr
La obesidad es uno de los factores de riesgo más ampliamente conocido y estudiado de la diabetes mellitus tipo 2 (DM2) ${ }^{1}$. Es parte importante del Síndrome $\mathbf{X}^{2}$, hoy conocido como síndrome metabólico, y redefinido hace más de 10 años por Reaven como la teoría de que la intolerancia a la glucosa, la hiperinsulinemia, la obesidad central, el aumento de la lipoproteina de muy baja densidad y disminusión de la de alta densidad, la hipertrigliceridemia y la hipertensión arterial esencial son consecuencia de la resistencia insulínica. Todos estos trastornos metabólicos se han relacionado con la DM2 y la enfermedad coronaria, esta última principal causa de muerte en Costa Rica. 
La obesidad es un trastorno nutricional que tiene una alta prevalencia en Costa Rica en adultos, adolescentes y niños. Los datos comparativos de las últimas dos encuestas nacionales de nutrición, demuestran que la prevalencia de la obesidad aumentó de un $34.6 \%$ en 1982 a un $45.9 \%$ en 1996 en mujeres de 20-44 años, y de $55.6 \%$ a $75 \%$ en mujeres de $45-59$ años. Entre las mujeres de 15-19 años ésta fue de un $23.3 \%$, y en escolares de la zona metropolitana fue del 20\%. ${ }^{3}$ Algunos estudios específicos de areas urbano marginales (zonas urbanas periféricas con bajo nivel socioeconómico) han demostrado que la obesidad también ahí es prevalente, como el realizado en el distrito de Damas del área de Desamparados 3 , en el cual la obesidad en escolares aumentó de un $14 \%$ en 1996 a un 22\% en 1999 (Ministerio de Salud C. R, datos no publicados). En los pacientes diabéticos la obesidad también es altamente prevalente, como se documenta en la literatura internacional ${ }^{4,5} \mathrm{y}$ en algunos estudios en Costa Rica ${ }^{6,7}$.

Lo anterior reafirma que la obesidad es una variable imprescindible y probablemente un pivote en el estudio de la historia "natural" de la de DM2 y de la intolerancia a la glucosa. Por eso debería ser considerada como una variable obligatoria a tratar en cualquier intervención de la DM2, ya sea en el campo preventivo o de su control metabólico.

Si queremos prevenir en el futuro una patología crónica como la DM2, uno de los retos del día de hoy es identificar cómo los genes y los factores ambientales interactúan para llevar a la obesidad a ciertos individuos. En el futuro cercano, la genética nos permitirá identificar aquellos individuos que tienen mayor probabilidad de desarrollar obesidad; sin embargo, es necesario continuar con la identificación de "predictores" metabólicos, clínicos, de comportamiento (hábitos) y/o ambientales de la obesidad y por ende de la DM2.

¿Existen indicadores metabólicos o clínicos que puedan identificar individuos en riesgo para desarrollar DM2 además de la obesidad?

¿Es la leptina un posible factor predictor de la DM2?

La teoría señala a una relación de la leptina con la obesidad y ésta a su vez ha sido asociada a la diabetes en modelos animales de obesidad.

En 1978 Coleman, ${ }^{8}$ y casi diez años más tarde, Hervey y colaboradores ${ }^{9}$, detectaron la presencia de un factor circulante que regulaba la magnitud de los depósitos de grasa del cuerpo y el balance energético. Coleman sugirió que los ratones OB/OB (modelo animal de obesidad genética) carecían de ese factor, en cambio el ratón $\mathrm{db} / \mathrm{db}$ producía dicho factor, pero no tenía respuesta a sus efectos. Recientemente se descubrió que este factor es una hormona llamada Leptina, que es producida por el gen ob adipocito-específico, primero en el gen $\mathrm{OB}$ en el ratón y luego su homólogo humano ${ }^{10}$. El ratón $\mathrm{OB} / \mathrm{OB}$ cepa mutante que carece de leptina es obeso e hiperfágico. La administración central o periférica de leptina recombinante a estos ratones determina en agudo una drástica reducción de la ingesta y en forma crónica conduce a una marcada disminución de peso. Esta respuesta implica la existencia de receptores cerebrales para leptina, localizados a nivel hipotalámico, involucrados en la regulación central de la ingesta y del balance energético ${ }^{11}$.

Como había sido sugerido por Coleman en sus estudios iniciales, el ratón db/db (ratón obeso y diabético) produce leptina pero tiene una insensibilidad hipotalámica a sus efectos, es decir un modelo de leptino-resistencia. En humanos, si bien recientemente se describieron casos de obesidad severa por ausencia de leptina ${ }^{12}$, este modelo de leptino-resistencia ${ }^{13}$ sería el ampliamente predominante, probablemente por defectos a nivel del receptor ${ }^{14}$.

En estudios recientes, los niveles plasmáticos de leptina han correlacionado con ciertos factores del Síndrome metabólico, incluyendo hipertensión esencial ${ }^{15}$, obesidad ${ }^{16,17,18}$, resistencia insulínica ${ }^{19}$ y lipoproteína de alta densidad ${ }^{20}$.

El presente estudio pretende iniciar la búsqueda de predictores de DM2 en escolares adolescentes de una zona urbano marginal del área metropolitana, determinando la prevalencia de la obesidad, la dislipidemia y los niveles de un marcador metabólico como es la leptina, bajo el proyecto de investigación "Factores de riesgo para Diabetes Mellitus Tipo 2 en escolares del Area 3 Desamparados".

\section{Material y métodos:}

Estudio transversal realizado durante el año 2001, a una población total de 747 escolares menores de 14.8 años de sexto grado de 12 Escuelas del Área de Desamparados 3. Previo consentimiento escrito de padres y escolares aprobado por el Comité de bioética de la Universidad de Costa Rica se midió peso y talla a toda la población; se obtuvo suero en ayunas de 12 horas en una muestra aleatoria de escolares para cuantificar colesterol total, HDL-colesterol, triglicéridos considerando una prevalencia de .3 de dislipidemia, con una diferencia máxima de .045 entre los resultados y $95 \%$ de confianza dando una $\mathrm{n}=261$; a ésta se le agregó un $10 \%$ más por posibles rechazos, resultando la muestra total en 287 . Para analizar la leptinemia se utilizó una submuestra aleatoria de 220 de la muestra para lípidos, por limitaciones presupuestarias.

Datos antropométricos: Según la Normativa de Atención Integral del Niño(a) en el escenario escolar, se aplicó en el Área de Desamparados 3 a todos los escolares sexto grado, una valoración del estado nutricional por medio de la medición del Indice de Masa Corpora( $1 \mathrm{MC}$ ) (peso / talla 2 ) según ANEXO No. 6 del Manual de Atención Integral de los niños en el escenario escolar. Esta valoración se realizó en los meses de junio y julio 2001.

Toma de la muestra de sangre, análisis químico clínico y de radioinmunoensayo: Los escolares se sangraron por punción venosa en brazo derecho, con un ayuno mínimo de 12 
horas, en sus escuelas a primera hora del día (7.a.m). Se obtuvo dos muestras de 3 cc cada una. Una se trasladó al Laboratorio Clínico de la Clínica Dr. Marcial Fallas para su proceso de centrifugación y medición de lípidos por medio de una técnica enzimática con analizador automático en el Equipo Targa BT 3000 y reactivos de la casa Wiener. La segunda muestra se llevó al Instituto de Investigaciónes en Salud, U.C.R en donde se centrifugó y congeló el suero a -70 grados hasta que se analizaron como un solo lote (todas las muestras en un mismo día, marzo 2001), por ser un método en el que se utiliza la radioactividad que es perecedera: Se utilizó el ensayo inmunoradiométrico (IRMA) de la casa Diagnostic Systems Labrotories Inc, el cual incluye controles de leptina humana de concentraciones conocidas.

Análisis de los datos: Estos fueron analizados con el programa de Excel de Windows, con métodos no paramétricos; para el análisis de la asociación de leptinemia con sexo y sobrepeso se utilizaron los métodos Kruskal-Wallis Test y Wilcoxon Rank Sum Test con un nivel de confianza del 95\%; para estudiar la asociación entre variables continuas (lípidos e IMC) y leptinemia, se calculó el coeficiente de correlación de Spearman con un nivel de significancia de $\mathrm{p}<0,05$.

\section{Resultados:}

\section{Niveles de leptina en sangre}

Los niveles de leptinemia mostraron una distribución no normal, por lo cual su análisis se realizó con métodos no paramétricos. Su rango varió desde una mínima de $1.0 \mathrm{ng} / \mathrm{ml}$ a un valor máximo de $42.8 \mathrm{ng} / \mathrm{ml}$ en hombres, y de $3.9 \mathrm{ng} / \mathrm{ml}$ a $65.2 \mathrm{ng} / \mathrm{ml}$ en mujeres. Cuadro No. 1. La comparación de cuartiles según sexo fue altamente significativa $(\mathrm{p}<0.0003)$ siendo mayor en las mujeres. Cuadro No. 2.

\section{Correlación de Leptinemia con Lípidos e índice de masa corporal}

La concentración de leptina en plasma presentó una correlación positiva aunque baja con el $\operatorname{IMC}$ ( $\mathrm{rs}=0.43$, $\mathrm{p}<5.7$ E-11) y los triglicéridos ( $r s=0.31, \mathrm{p}<4.3 \mathrm{E}-06)$. El HDL-colesterol presentó también una baja correlación pero negativamente $(\mathrm{rs}=0.20, \mathrm{p}<0.002)$. No se encontró correlación con colesterol total,ni LDL-colesterol. Al desglosar los datos por sexo, esta correlación positiva con el IMC se presentó sobretodo en el sexo femenino ( $\mathrm{rs}=0.59, \mathrm{p}<1.05$ E-12) y ésta fue moderada. El comportamiento de los triglicéridos fue similar en ambos sexos, no así el del HDL, en donde la correlación negativa solo se presentó en las mujeres ( rs $=0.40, \mathrm{p}<5.1 \mathrm{E}-06)$, pero también fue baja. Cuadro No 3 , Gráfico No 1 y 2).

\section{Relación de Leptinemia con el sobrepeso}

Al comparar los cuartiles de los valores de leptina contra el estado nutricional de los niños, se encontró diferencias significativas para ambos sexos entre las categorías de normal y sobrepeso $(\mathrm{p}<0.01)$ delgado y sobrepeso $(<0.05)$, no así entre delgado y normal. Cuadro No 4.

\section{Discusión}

La obesidad se comporta como una epidemia en el mundo. En la última encuesta nacional de Costa Rica se demostró que más del $50 \%$ de las mujeres adultas eran obesas y que la tendencia en los últimos años tiende hacia el aumento de su prevalencia. Los niños y los adolescentes no escapan de esta tendencia con una prevalencia de 14-20\%.

En lo últimos años se ha logrado un gran progreso en la identificación de genes que están involucrados en la regulación del peso y de la obesidad. Se han encontrado más de 200 loci que podrían estar relacionados con el peso y/o la obesidad. En roedores se han identificado y caracterizado varios modelos de obesidad que comprometen un solo gen. Existen alrededor de 25 casos de obesidad en humanos que se han explicado por mutaciones genéticas.

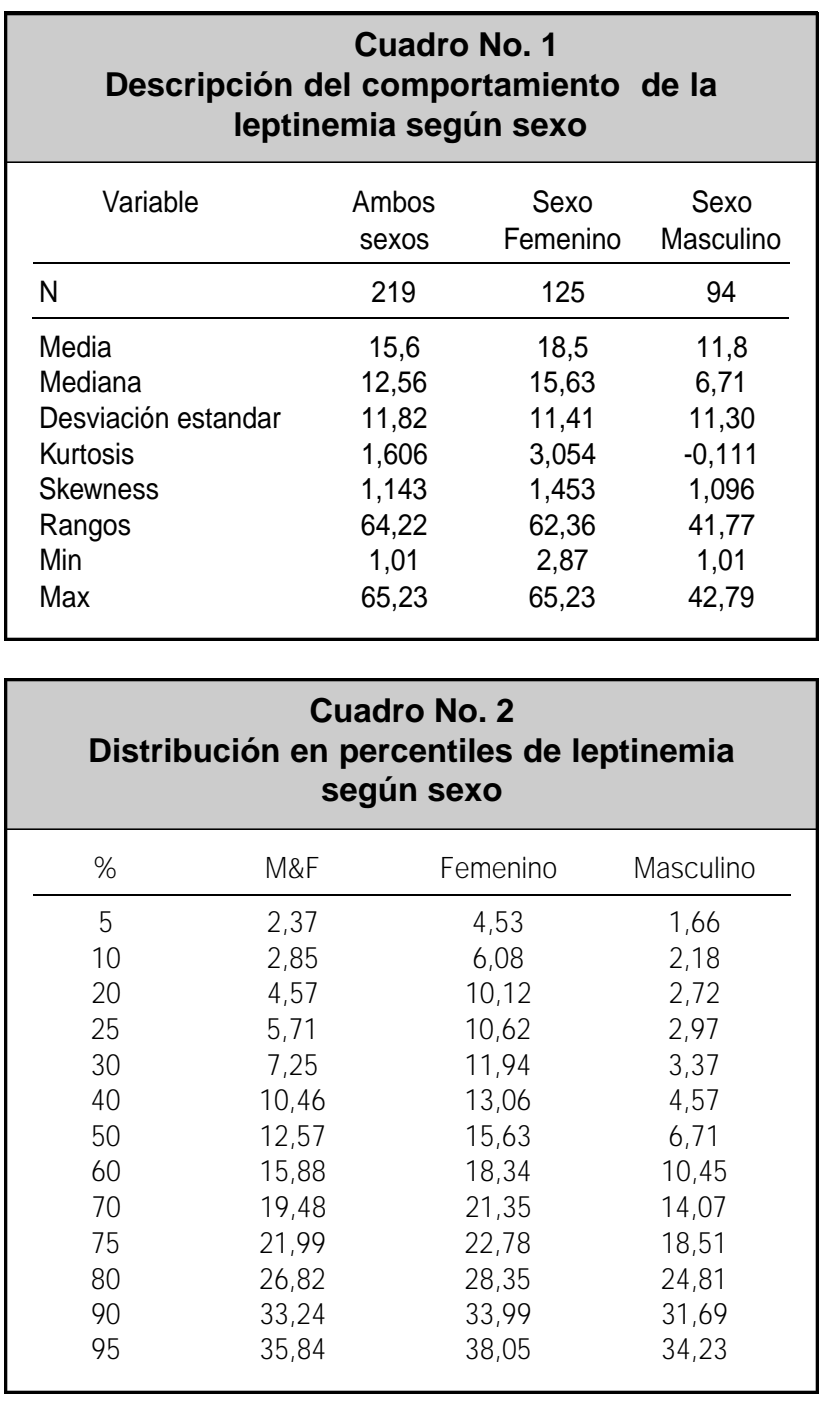


No hay duda que la investigación en genética ha permitido dilucidar mucho de la fisiología que permite que la obesidad se desarrolle, pero la obesidad es un estado poligénico, involucrando muchos genes. Aun así, aunque la genética ayuda a entender que pasa con la obesidad, el aumento tan dramático de ésta, tiene que ser resultado de factores coadyuvantes ambientales.

La leptina, descrita recientemente, es una hormona producto del gen ob, y se cree que juega un papel preponderante en la regulación de la cantidad de adiposidad del cuerpo. ${ }^{21}$ Además de esta función, parece ser un componente de varios enlaces de retroalimentación en los ejes hipotálamo, pituitaria y órganos endocrinos y también es un factor estimulante y de retroalimentación en el eje reproductivo.

Siendo la adolescencia un período de grandes cambios metabólicos y hormonales, los niveles de leptina y otros parámetros hormonales parecen tener relación importante con los cambios mayores en la composición corporal como en su función gonadal en este período.

El presente estudio, es un primer intento para describir el comportamiento de esta hormona en adolescentes de una comunidad urbano-marginal del área metropolitana. Los resultados encontrados fueron similares a los descritos en otros estudios de la literatura internacional llevados acabo en países sobretodo desarrollados.

Los valores de leptinemia en los escolares fueron más altos en el sexo femenino, con una relación de 2:1 al sexo mascu-

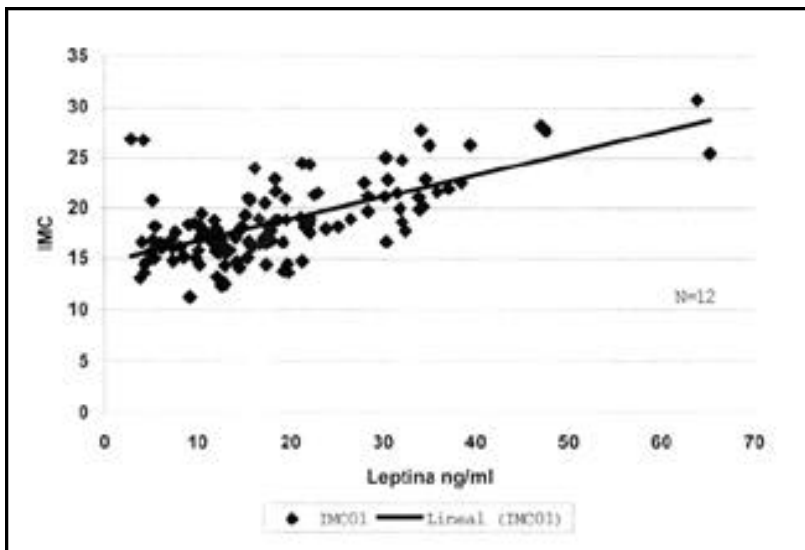

Gráfico 1: Correlación Leptina / IMC. Sexo Femenino.

lino. Este comportamiento también se describe en otros estudios en países desarrollados ${ }^{18,22,23,24}$ en los cuales se asoció un posible papel de la testosterona como responsable de esta diferencia entre sexos. En un estudio en Indiana, U.S.A. en niños y adolescentes (9-20años), se encontró que las mujeres tenían niveles de leptinemia 2.15 veces más elevados que los hombres y una relación decreciente con la edad en los hombres pero no en mujeres. También se encontró una fuerte relación inversa con los niveles de testosterona. ${ }^{22}$

Otro estudio en el Reino Unido presentó lo mismo: concentraciones similares de leptina en ambos sexos en los años prepuberales, con un aumento paralelo en el inicio de la pubertad, para decrecer en el sexo masculino después de la pubertad, mientras en las mujeres los niveles se mantenían constantes ${ }^{23}$. En un estudio en Alemania (edad 6-20años) también se encontró esta misma relación de disminución de leptina en hombres después de la pubertad ${ }^{18} \mathrm{y}$ la relación inversa de testosterona/leptina, con la consecuente diferencia significativa entre sexos. En Asia, China, también se describe este mismo patrón en adolescentes chinos (14-16 años), con niveles de leptinemia mayores en el sexo femenino que en el masculino $(18.53 \pm 1.41$ vs. $6.33 \pm 1.79$ microgramos $/ \mathrm{L}) .{ }^{24}$

Esta relación con los niveles de testosterona podría ser la razón de la disminución de leptina en el sexo masculino con relación a la edad. Esto plantea la posibilidad de que la testosterona en los hombres pueda tener un efecto supresivo de la producción de leptina en el adipocito.

110 AMC, Julio-Setiembre 2003, vol 45 (3) 


\begin{tabular}{|lcccc|}
\hline \multicolumn{5}{c|}{ Cuadro No. 4 } \\
Relación de leptina con sobrepeso \\
\hline Hombre & & & \\
\hline Leptina & Delgado & Normal & Sobrepeso & Total \\
& $\mathrm{n}(\%)$ & $\mathrm{n}(\%)$ & $\mathrm{n}(\%)$ & $\mathrm{n}(100 \%)$ \\
1 cuartil & $1(8.3)$ & $11(91.7)$ & 0 & 12 \\
2 cuartil & $6(18.8)$ & $24(75.0)$ & $2(6.3)$ & 32 \\
3 cuartil & $4(10.5)$ & $24(63.2)$ & $10(26.3)$ & 38 \\
4 cuartil & $1(8.3)$ & $7(58.3)$ & $4(33.3)$ & 12 \\
& & & & \\
Mujer & & & & \\
\hline Leptina & Delgado & Normal & Sobrepeso & Total \\
& $\mathrm{n}(\%)$ & $\mathrm{n}(\%)$ & $\mathrm{n}(\%)$ & $\mathrm{n}(100 \%)$ \\
1 cuartil & $4(20.0)$ & $14(70.0)$ & $2(10.0)$ & 20 \\
2 cuartil & $8(20.5)$ & $31(79.5)$ & $0(0.0)$ & 39 \\
3 cuartil & $5(12.8)$ & $31(79.5)$ & $3(7.7)$ & 39 \\
4 cuartil & 0 & $14(51.9)$ & $13(48.1)$ & 27 \\
\hline
\end{tabular}

El presente estudio no incluyó la evaluación del desarrollo sexual ni la interacción con otras hormonas como la testosterona, por lo que no se pudo evaluar la asociación de la leptina con la función gonadal. Se considera importante que estudios futuros en adolescentes se incluyan niveles de testosterona y la prueba de Tanner, para analizar los componentes hormonales de este momento del ciclo de vida tan complejo.

Por otro lado, la leptina también ha tenido correlación con varios componentes del síndrome metabólico, entre ellos la obesidad y la dislipidemia (triglicéridos altos y HDL-colesterol bajo). En el análisis de los resultados de los lípidos y el IMC con la leptina en este grupo de escolares se encontró una correlación positiva aunque débil con el IMC y triglicéridos y negativa con el HDL-col, sobretodo en mujeres.

También se demostró en ambos sexos una asociación significativa con el estado nutricional: delgado/sobrepeso y normal/sobrepeso, demostrando lo ya conocido de que la leptina aumenta en proporción a la adiposidad. ${ }^{25,17}$

Los hallazgos anteriores también han sido descritos en otros estudios realizados en niños y/o adolescentes en otros países.

Por ejemplo, en un estudio en España, en niños prepuberales, la leptina correlacionó significativamente con el IMC ( $\mathrm{r}=.57$, $\mathrm{p}<0.001)$ y triglicéridos $(\mathrm{r}=.31, \mathrm{p}<0.007)$, pero no con el HDL-colesterol. Pero, en el análisis factorial de las concentraciones de leptina con varios conglomerados de factores, si presentó relación estadística con el factor compuesto por varias variables relevantes del síndrome metabólico, formado por valores altos en la prueba de intolerancia a la glucosa intravenosa/el factor de obesidad central y un factor compuesto por la relación triglicéridos altos/HDL-col bajo. ${ }^{26}$

En el mismo estudio citado anteriormente realizado en China, se encontró diferencias significativas entre obesos y no obesos, para leptinemia y triglicéridos y HDL colesterol,con valores más altos en los obesos, para los primeros dos parámetros y más bajos para el tercero en los adolescentes.

Como conclusión,el encontrar similitud en el comportamiento del perfil de leptinemia en los adolescentes de este estudio y de otros de diferente afinidad biológica, desarrollo social y área geografica, permite suponer que esta hormona tiene un patrón que no varía por éstas variables.

En consecuencia, los niveles de leptinemia en adolescentes se caracterizan por:

- Ser mayor en mujeres que en hombres

- Correlacionar positivamente con IMC, más en mujeres

- Correlacionar negativamente con HDL

- Presentar diferencias significativas entre adolescentes con y sin sobrepeso

\section{Abstract}

Introduction: The hormone called Leptine has been associated with several factors of the Metabolic Syndrome, including hypertension, obesity, insulin resistance and high density lipoprotein, not only in adults but also in adolescents. In Costa Rica the levels of leptinemia in adolescents and its relationship with obesity and lípids is unknown.

Objective: To describe the leptinemia levels in sixth graders in an urban- marginal area, and its association with lipids and overweight.

Material And Methods: Transversal study was carried on in the year 2001, with a representive sample sixth graders of 12 Schools of the Area Desamparados $3(n=220)$. Previous written consent of parents and scholars, weight and height was measured and a 12 hour plasma sample was taken to quantify leptine (IRMA,) total cholesterol, HDL- colesterol and triglicerides; the LDL-col was calculated.

Results: The leptinemia levels did not follow a normal distribution, so non-parametric analysis was used. Its range varied from a minimum value of $1.0 \mathrm{ng} / \mathrm{ml}$ to a maximum value of $42.8 \mathrm{ng} / \mathrm{ml}$ in males, and from $3.9 \mathrm{ng} / \mathrm{ml}$ to $65.2 \mathrm{ng} / \mathrm{ml}$ in $\mathrm{fe}-$ males. The comparison of quartiles by sex was highly significant ( $p<0.0003$ ); having higher levels in females. The plasma leptine concentration showed a positive correlation with the Body Mass Index (BMI) (rs=0.43, p<5.7E-11) and triglicerides ( $\mathrm{rs}=0.31, \mathrm{p}<4.3 \mathrm{E}-06$ ), but a negative correlation with HDL- cholesterol (rs -0.20, p <0.002); there was no correlation with total cholesterol or LDL- cholesterol. When the correlation was done by sex, the positive correlation was given mainly by the feminine sex (rs 0.59, p <1.05E-12) and its was moderate. The triglicéridos behavior was similar in both sexes, not so with the HDL, where the negative correlation was only found in females (rs $-0.40 \mathrm{p}<5.1 \mathrm{E}-06$ ), but it was weak. When the quartiles of leptine were compared 
with the children's nutritional status a significant differences was found in both sexes between the categories of normal and overweight $(\mathrm{p}<0.01)$, and between slender and overweight $(<0.05)$, but not between the slender and normal categories.

Conclusion: The leptinemia values of the scholars were higher in the feminine sex, with a relation of $2: 1$. The correlation of this variable even though weak, was positive for BMI and negative with HDL-cholesterol, especially in the female adolescents. Significant differences were found between the slender and normal adolescentes and those that were overweight. The results of this study are coincident with other studies in the international literature.

\section{Referencias}

1. Bray G.A. Health hazards of obesity. Endocrinol Metab Clin North Amer 1996; 25:907-919..

2. Reaven GM. Role of insulin resistance in human disease. Diabetes 1988;37:1595-607

3. Ministerio de Salud, Fascículo 1. Antropometría,Encuesta Nacional de Nutrición, Costa Rica 1996

4. Gumbiner B. The treatment of obesity in type 2 diabetes mellitus Prim Care. 1999 Dec;26(4):869-83. .

5. Campbell L, Rossner S. Management of obesity in patients with Type 2 diabetes. Diabet Med. 2001 May; 18(5):345-54.

6. Jiménez MF y Ruíz L. Niveles de glicemia y de hemoglobina glicosilada en un grupo de diabéticos nicoyanos. AMC 2001 ; 43 (suplemento 1$): 15$

7. Laclé A. Perfil del Paciente Diabético Tipo 2 de 55 y Más Años,de una Clínica Periférica de la Caja Costarricense de Seguro Social. AMC 1999; 41: 46-51.

8. Coleman DL. Obesity and diabetes: Two mutant genes causing diabetes-obesity syndromes in mice. Diabetología 1978; 14:141-8.

9. Harris RB, Hervey E, Hervey G R. Body composition of lean and obese Zucker rats in parabiosis. Int J Obesity 1987; 11:275-83.

10. Zhang Y, Proenca R, Maffei M, Barone M, Leopold L, Friedman JM. Positional cloning of the mouse obese gene and its human homologue. Nature 1994; 372:425-32.

11. Halaas J, Gajiwala KS, Maffei M,Cohen SL, Chait BT, Rabinowitz D, Lallone RL, Burley SK, Friedman JM. Weight reducing effects of the plasma protein encoded by the obese gene. Science 1995; 269:543-6.

12. Montague CT, Farooqi IS, Whitehead JP, Soos MA, Rav H, Wareham WJ et al. Congenital leptin deficiency is associated with severe earlyonset obesity in humans. Nature 1997; 387:903-8.

13. Caro JR,Sinja MK, Kolaczynski JW, Zhang PL,Cosindine RB. Leptin: The tale of an obesity gene. Diabetes 1996; 45:1455-61.

14. Lönnqvist F, Arner P, Nordfors L, Shalling M. Overexpression of the obese gene mRNA in human obesity. J Clin Invest 1995; 95:2986-8

15. Agata J.,Masuda A, Takada M,Higashiura K, Murakami H,Miyazaki Y, Shimamoto K.High plasma inunoreactive leptin level in essential hypertension. Am J. Hypertens 1997; 10:117-4

16. Bray G, York D. Leptin and clinical medicine. A new piece in the puzzle of obesity. J. Clin Endocrinol Metab 1997; 82:27771-6

17. Dagogo_-Jack, S. Fanelli C, Parmore D, Brothers J, Landt M,. Palsma Leptin and Insulin relationships in Obese and Nonobese Humans ,Diabetes $1996: 45$ : 695-98
18. Blum,WF, Englaro P, Hanitsch S, Juul A,Hertel NT, Muller J, Skakkebaek NE, et al. Plasma leptin in Healthy Children and Adolescent:Dependence on Body Mas Index, bode Fat,Pubertal Stage and Testosterone. J Clin. Endocr and Metab 1997; 83:2904

19. de Courten M, Zimmet P, Hodge A, Collins V, Nicolson M,Staten M, et al . Hyperleptinemia: the missing link in the metabolic syndrome? Diabet Med 1997;14:200-8

20. Rainwater DL, Comuzzie AG, VandeBerg J, Mahney MC, Blangero J. Serum leptin levels are independently correlated with two measures of HDL. Atherosclerosis 1997; 132: 237-43

21. Auwerx J, Staels B. Leptin. Lancet 1998 ; 351 : 737-42

22. Ambrosius WT; Compton JA; Bowsher RR; Pratt JH, Relation of race, age, and sex hormone differences to serum leptin concentrations in children and adolescents Horm Res 1998;49(5):240-6

23. Clayton PE, Gill MS, Hall CM, Tillmann V, Whatmore AJ, Price DA, Serum leptin through childhood and adolescence, Clin Endocrinol 1997;46(6):727-33

24. Zhang J, Gao Y, Guo X, Dong A. . The changes in plasma letpin,insulin and proinsulin levels in obese adolescents Zhonghua Nei Ke Za Zhi 2002 41(4):221-3

25. Considine R, Sinha M, Heiman M, Kriacinus A, Stephens T, Nyce M, et al, Seum Immunoreactive concentrations in normal weight and obese humans,N Engl J Med 1996,334 (5) : 292-293

26. Valle M, Gascon F, Maros R, Ruz FJ, Bermudo F, Morales R, Canete R. Metabolic cardiovascular syndrome in obese prepubertral children; the role of high fasting insulin levels. Metabolismo 2002, , 51(4):423-8

\section{AMC, Julio-Setiembre 2003, vol 45 (3)}

